\title{
LOSSY COMPRESSION OF VOLUMETRIC MEDICAL IMAGES WITH 3D DEAD ZONE LATTICE VECTOR QUANTIZATION
}

\author{
Yann Gaudeau, Jean-Marie Moureaux \\ CRAN - CNRS (UMR 7039) Nancy-University, \\ Faculté des Sciences et Techniques, BP 239, \\ F-54506 Vandœuvre-lès-Nancy Cedex \\ France
}

\begin{abstract}
This paper presents a new lossy coding scheme based on 3D Wavelet Transform and Lattice Vector Quantization for volumetric medical images. The main contribution of this work is the design of a new codebook enclosing a multidimensional dead zone during the quantization step which enables to better account correlations between neighbour voxels. Furthermore, we present an efficient rate-distortion model to simplify the bit allocation procedure for our intra-band scheme. Our algorithm has been evaluated on several CT an MR image volumes. At high compression ratios, we show that it can outperform the best existing methods in terms of rate-distortion tradeoff. In addition, our method better preserves details and produces thus reconstructed images less blurred than the well-known 3D SPIHT algorithm which stands for a reference.
\end{abstract}

Index Terms - Lossy Compression, volumetric medical images, 3D wavelet transform, 3D Dead Zone Lattice Vector Quantization.

\section{INTRODUCTION}

Most of the current medical imaging techniques produce three-dimensional data. Some of them are intrinsically volumetric, like Positron Emission Tomography (PET), Computerized Tomography (CT), Magnetic Resonance (MR), 3D ultrasound, while others describe the temporal evolution of a dynamic phenomenon as a sequence of 2D images (they are more properly labelled as $2 \mathrm{D}+$ time). The huge amount of data generated every day in the hospital environment yields compression to be unavoidable for efficient storage and transmission purposes.

Compression schemes can be divided into two groups: lossless compression and lossy compression. Nowadays, compression methods used in medical applications are most of the time lossless methods in order to preserve the data integrity and to facilitate thus a true diagnosis. However, lossless coding does not permit to reach high compression ratios. Thus, most of applications (e-g: telemedecine, fast searching and browsing of medical volumetric data) suffer from this limitation. For this kind of applications, lossy compression seems to be an appropriate alternative. Furthermore, in many applications like for example computer aided detection (CAD) [1], it has been shown that a balance between data compression and data fidelity could be achieved [2]

A volumetric medical image is a three-dimensional (3D) image data set which can be considered as a sequence of two-dimensional (2-D) images (or slices). A direct way to perform compression on it is to straightforwardly apply a two dimensional compression algorithm to each slice independently. However, the slices are generally highly correlated with one another. Thus, the basic idea of the 3D medical image compression algorithms is to take advantage of the correlation among the data samples in the three dimensional space to improve compression performances. The most approaches combine a threedimensional space decorrelating transform with the extension of a coding algorithm that has proven to be effective on 2D images [3]. For example, Catin et al [4] were the first, to our knowledge, to compress volumetric medical images with Lattice Vector Quantization (LVQ), while many works had been done before on LVQ [5] [6] in the field of 2D real life images.

In this paper, we propose the design of a new entropycoded LVQ codebook enclosing a multidimensional dead zone to encode the coefficients of a 3D Dyadic Wavelet Transform (3D DWT) which is known to be one of the most efficient decorrelator for 3D medical images [3] [4].

One of the main advantages of the proposed scheme called 3D Dead Zone Lattice Vector Quantization (3D DZLVQ)- is its ability to remove non significant vectors while quantizing more accurately significant ones, which yields a significant improvement of the overall ratedistortion trade-off. Moreover, we introduce an efficient bit rate allocation procedure based on the approximation of the rate distortion (R-D) functions by an exponential model. Numerical and visual results produced by 3D DZLVQ on MRI and CT images are promising at low rates by comparison with a set of the best 3D coders published in the literature. 


\section{3D DEAD ZONE LATTICE VECTOR QUANTIZATION}

The whole lossy compression scheme we propose is represented in Figure 1. The coefficients produced by the 3D DWT within each subband are scaled and quantized by the 3D DZLVQ algorithm in an intra-band framework (dependencies between resolutions are not taken into account). The main advantage of LVQ-based methods is their low complexity since there is no need to construct or store the codebook. Quantized vectors $Y$ are finally encoded by using an efficient prefix code which associates to $Y$ a unique pair (e,pos), where $e=\|Y\|_{1}$ and pos stands for the position of $Y$ on the shell of radius $e$. For efficiency in terms of bit rate, $e$ is encoded using entropy coding while pos is fixed-length coded [7]. The bit rate related to a subband is then:

$$
R=-\sum_{r=0}^{r_{T}} P(e=r)\left\{\log _{2} P(e=r)-\left\lceil\log _{2} N(r)\right\rceil\right\} \text { bits/vector }
$$

where $N(r)$ is the population of the shell of radius $r$ (i.e. the number of codebook vectors whose norm ${ }^{1}$ is equal to $r$ ), $P$ the discrete law of the radius and $r_{t}$ the truncation radius of the codebook.

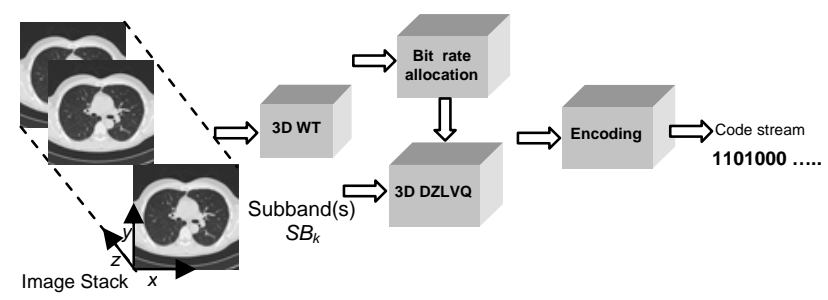

Figure 1: Proposed whole lossy compression scheme.

Here we propose to decrease $R$ at a fixed distortion $D$ (or equivalently to decrease $D$ at a fixed rate $R$ ) by designing a multidimensional dead zone of radius $R_{D Z}$ within the codebook. Our scheme allows to threshold non significant source vectors according to a criterion based on their norm, while putting more bits on significant ones. It can efficiently apply to medical images in the 3D wavelet domain which often contain huge non-significant areas (for instance the areas where no anatomy is imaged).

Furthermore, the norm of vectors is a good measure of the local activity in the case of correlated and sparse source samples like wavelet coefficients under the condition of vectors oriented along the direction of the details of the corresponding subband. To discuss this issue, let us make a comparison between the histograms of the norm of three kind of vectors: Figures 2 (A) to (C)

\footnotetext{
${ }^{1}$ In the whole paper the norm stands for the $L^{1}$ norm.
}

correspond to the $\mathrm{LHL}_{3}^{2}$ subband (vertical details + low pass filtering along $\mathrm{z}$ ) of liver_t2 image (see Table 1 ) for three different vector orientations: vertical $(8 \times 1 \times 1)$, horizontal $(1 \times 8 \times 1)$ and cubic $(2 \times 2 \times 2)$ respectively. Distribution (A) is more picked than (B) since an orientation along the direction of wavelet details allows to better capture clusters of significant coefficients and to increase the number of vectors around zero. This point shows that for coding efficiency purposes one should not privilege vectors orthogonal to the direction of the 2D wavelet subband details. Moreover, as we perform a 3D DWT, additional correlation exists along the temporal direction (z) as it is shown in Figure 2(C) with the cubic orientation of vectors. This last one represents actually a good compromise for capturing clusters in the three directions in all the subbands. Logically, the cubic orientation gives the best R-D function of 3D DZLVQ for all the subbands. So, will be used in our work.
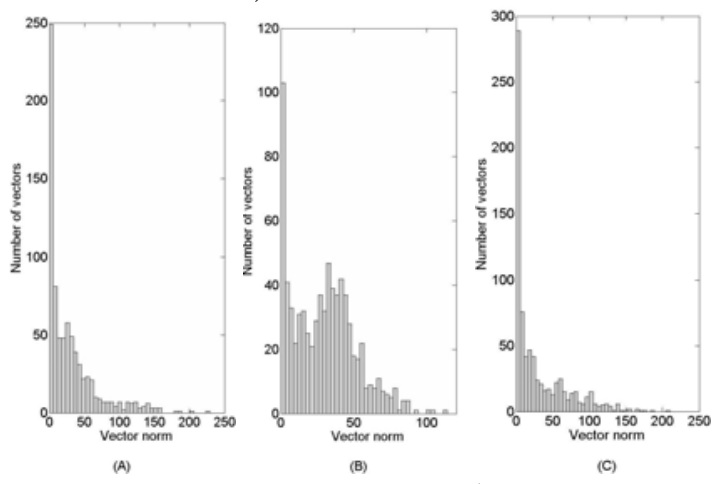

Figure 2: Histograms of the $L^{1}$ norm of the LHL3 subband of Liver t2: 8 × 1 x 1 vectors (A), 1 x 8 x 1 vectors (B), $2 \times 2 \times 2$ vectors (C).

In the proposed framework, according to the vector dead zone radius $R_{D Z}$, the quantization process of any source vector $X$ becomes the following (see Figure 3):

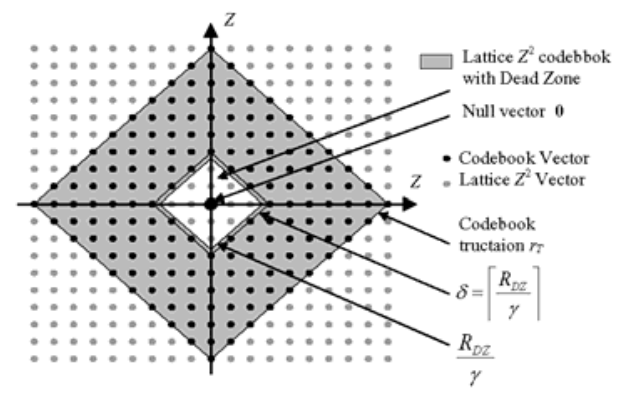

Figure3: 3D DZLVQ codebook for vectors of size

If $\|X\|_{1} \leq R_{D Z}, X$ is replaced by the null vector $\mathbf{0}$.

If $\|X\|_{1}>R_{D Z}, X$ is scaled by $\gamma$ (the scaling factor) and quantized by usual fast lattice quantization algorithms [7]. Note that the parameters $\left(\gamma, R_{D Z}\right)$ enable to tune the tradeoff between rate and distortion.

\footnotetext{
${ }^{2} \mathrm{~L}$ and $\mathrm{H}$ mean low pass and high pass filtering respectively. The filtering direction follows the order $x, y$ and $z$. The final number indicates the level decomposition
} 


\section{BIT RATE ALLOCATION PROBELM}

As, we use an intra-band approach to quantize and to encode the wavelet coefficients, it is necessary to implement a rate allocation algorithm to assign a bit rate to each subband $S B_{k}$ of the multiresolution decomposition, while minimizing the overall distortion, under the constraint to reach the total bit rate. This is a difficult problem (especially for volumetric images) which can be solved by lagrangian multiplicators based methods at the price of a high computational complexity. A lot of effort has been done for years by many researchers to develop analytical rate-distortion models dedicated to wavelet coefficients to decrease the complexity of the allocation procedure. However, these models are generally built on the i.i.d. hypothesis [6] [8] while block based methods (like LVQ) could take advantage of non i.i.d. properties of real data such as wavelet coefficients. In the 3D DZLVQ case, this drawback can be circumvented. Indeed, as 3D DZLVQ increases the amount of null vectors, it modifies the shape of the R-D function in the low rates range in a way that it can be modeled by a single exponential function $D(R)=C e^{-a R}$ with $C$ and $a$ the parameters of the model.

These parameters can be computed using a simple linear regression. Let $L$ be the number of $R-D$ points used in the regression and $d_{k}=D\left(R_{k}\right), k=1, \ldots, L$ be the distortion value associated to the bit rate $R_{k}$ of subband $S B_{k}$. The parameters of our model are then:

$$
\begin{gathered}
C=\exp \left(\frac{L \sum_{k=1}^{L} R_{k} d_{k}-\sum_{k=1}^{L} R_{k} \sum_{k=1}^{L} d_{k}}{L \sum_{k=1}^{L} R_{k}^{2}-\left(\sum_{k=1}^{L} R_{k}\right)^{2}}\right) \\
a=-\frac{1}{L}\left(\sum_{k=1}^{L} d_{k}-\ln (C) \sum_{k=1}^{L} R_{k}\right)
\end{gathered}
$$

Practically, we can show that only three R-D points are necessary to compute $C$ and $a$. Figure 4 permits to compare the experimental R-D function of $\mathrm{LHL}_{2}$ subband of Liver_t2 image and the approximation given by our model. As we can see, the proposed model is accurate.

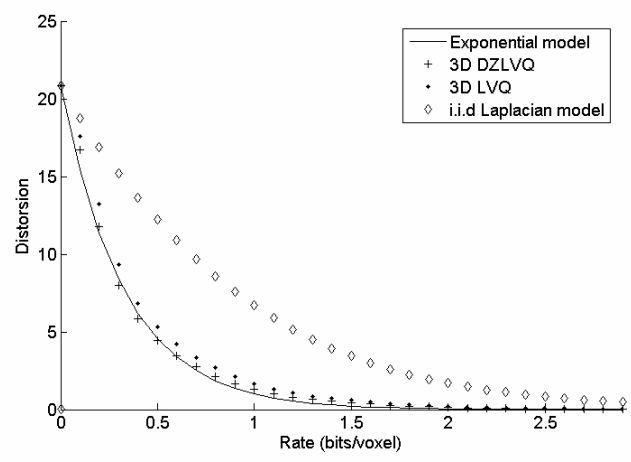

Figure 4: Comparison of R-D functions obtained with LVQ, 3D DZLVQ, i.i.d Laplacian model and proposed exponential model - LHL2 subband of Liver $t 2$
Finally, it offers substantial reduction of the computational complexity of rate allocation as it leads to an analytical solution to this problem. The interested reader may consult the reference [9] related to the 2D case for more precision.

\section{EXPERIMENTAL RESULTS}

In order to give a fair comparison with existing works, we have tested our 3D DZLVQ algorithm for lossy compression on the 8-bit CT and MR volumetric medical image data set used in Bilgin et al's work [3]. Table 1 shows the description of these images. The first slices of each data set are shown in Figure 5.

\begin{tabular}{c|c|c|c|c}
\hline & History & File name & Voxel size(mm) & Volume size \\
\hline CT & tripod fracture & Skull & $0.7 \times 0.7 \times 2$ & $256 \times 256 \times 128$ \\
& scaphoid fracture & Wrist & $0.17 \times 0.17 \times 2$ & $256 \times 256 \times 176$ \\
& carotid dissection & Carotid & $0.25 \times 0.25 \times 1$ & $256 \times 256 \times 64$ \\
& Apert's syndrome & Aperts & $0.35 \times 0.35 \times 2$ & $256 \times 256 \times 96$ \\
\hline MR & Normal & Liver_t1 & $1.45 \times 1.45 \times 5$ & $256 \times 256 \times 48$ \\
& Normal & Liver_t2 & $1.37 \times 1.37 \times 5$ & $256 \times 256 \times 48$ \\
& Left Exopthalmos & Sag_head & $0.98 \times 0.98 \times 3$ & $256 \times 256 \times 48$ \\
& Heart disease & Pad_chest & $0.78 \times 0.78 \times 5$ & $256 \times 256 \times 64$ \\
\hline
\end{tabular}

Table 1: Description of the data sets in our experiments.

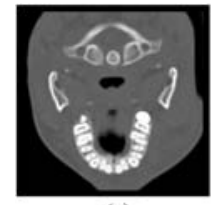

(a)

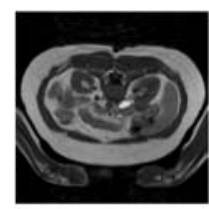

(e)

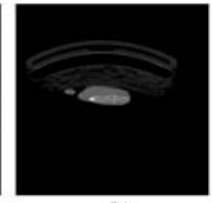

(b)

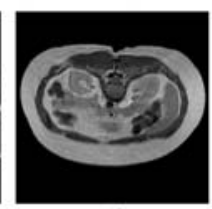

(f)

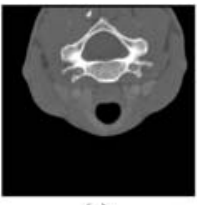

(c)

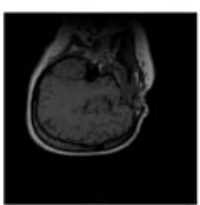

(g)

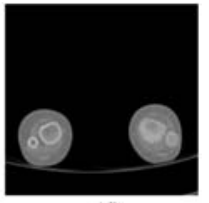

(d)

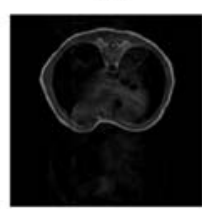

(h)
Figure 5: Caption of volumetric medical images : First slice of each data set (a) Skull (b) Wrist (c) Carotid (d) Aperts (e) Liver_t1 (f) Liver_t2e1 (g) Sag_head (h) Ped_chest

First, we compare the compression performances of our method with those of both classical 3D LVQ [4] and 3DSPIHT [10] [11]. All the images have been compressed by the three methods at rates varying from 0.05 to 1 bit/voxel (bpv). We have used the well-known 9.7 floating-point filter [6] with a four-level 3D DWT or a three-level 3D DWT when the resolution was limited along the $z$ axis $\left(T_{Z}<64\right)$. Furthermore, we have designed a pyramidal codebook whose dead zone and scaling factors (within each subband) have been chosen to optimize the overall rate-distortion trade-off.

Table 2 shows that at a low rate $(0.1 \mathrm{bpv}) 3 \mathrm{D}$ DZLVQ outperforms 3D LVQ and 3D SPIHT, in terms of PSNR, except for Wrist and Aperts images. Note that tests at other low rates (lower than $0.5 \mathrm{bpv}$ for Skull and $0.35 \mathrm{bpv}$ for the remaining images) have lead to the same kind of results. 3D SPIHT is better at high rates (in terms of 
PSNR) since the efficiency of the vector dead zone naturally decreases at such rates.

\begin{tabular}{|c|c|c|c|}
\hline & 3D DZLVQ & 3D SPIHT & 3D LVQ \\
\hline Skull & $\mathbf{3 4 . 6 9} \mathbf{~ d B}$ & $33.98 \mathrm{~dB}$ & $33,46 \mathrm{~dB}$ \\
\hline Wrist & $43.63 \mathrm{~dB}$ & $\mathbf{4 4 . 9 5} \mathbf{~ d B}$ & $43.29 \mathrm{~dB}$ \\
\hline Carotid & $\mathbf{4 1 . 0 8 ~ d B}$ & $40.51 \mathrm{~dB}$ & $40.24 \mathrm{~dB}$ \\
\hline Aperts & $46.98 \mathrm{~dB}$ & $\mathbf{4 7 . 4 1} \mathbf{~ d B}$ & $46.66 \mathrm{~dB}$ \\
\hline Liver_t1 & $\mathbf{3 8 . 8 4} \mathbf{~ d B}$ & $38.37 \mathrm{~dB}$ & $38.02 \mathrm{~dB}$ \\
\hline Liver_t2el & $\mathbf{3 6 . 6 1 ~ d B}$ & $36.31 \mathrm{~dB}$ & $36.12 \mathrm{~dB}$ \\
\hline Sag_Head & $\mathbf{3 9 . 0 3 ~ d B}$ & $38.76 \mathrm{~dB}$ & $38.55 \mathrm{~dB}$ \\
\hline Pad_chest & $\mathbf{4 2 . 9 8 ~ d B}$ & $42.97 \mathrm{~dB}$ & $42.18 \mathrm{~dB}$ \\
\hline
\end{tabular}

Table2: Mean PSNR (dB) Comparison between 3D SPIHT, 3D LVQ and 3D DZLVQ at.0.1 bits/voxel (bpv) on the 8 database images detailed in Table

Furthermore, table 3 summarizes some results achieved by other 3D methods on the Skull image which is abundantly used in the literature [3], [10]-[12]. 3D EZW [3] is a recent extension of well-known EZW algorithm. $3 \mathrm{D}$ ESCOT is a $3 \mathrm{D}$ version of EBCOT algorithm using a uses a $(2+2,2)$ filter with a four-level integer wavelet packet transform and a very sophisticated contextmodeling [12]. Table 3 confirms the superiority of the proposed algorithm in terms of PSNR at low rates.

\begin{tabular}{|l|l|l|l|l|}
\hline & $3 \mathrm{D}$ SPIHT & 3D DZLVQ & 3D EZW & 3D ESCOT \\
\hline $0.5 \mathrm{bpv}$ & $44.11 \mathrm{~dB}$ & $\mathbf{4 4 . 1 4} \mathbf{~ d B}$ & $39.82 \mathrm{~dB}$ & $43,82 \mathrm{~dB}$ \\
\hline $0.1 \mathrm{bpv}$ & $33.98 \mathrm{~dB}$ & $\mathbf{3 4 . 6 9} \mathbf{~ d B}$ & $31.68 \mathrm{~dB}$ & $34,68 \mathrm{~dB}$ \\
\hline
\end{tabular}

Table3: PSNR (dB) Comparison on 128 slice Skull between 3D SPIHT, 3D ESCOT, 3D CB-EZW, 3D LVQ and 3D DZLVQ

Finally, Figure 6 provides a reconstructed slice of the Skull image, after a compression at $0.1 \mathrm{bpv}$ on the whole image by both 3D DZLVQ and 3D SPIHT. We can see that at this rate, our method seems to better preserve details, producing thus an image less blurred than 3D SPIHT. Note that at high rates, it seems more difficult to note differences between the methods.

\section{DISCUSSION}

We have presented here a new lossy compression method for volumetric medical images. It is based on a $3 \mathrm{D}$ wavelet transform followed by a 3D dead zone lattice vector quantizer. Our scheme exploits intra-band dependencies, contrary to methods like 3D SPIHT which take into account dependencies existing between wavelet coefficients at the different resolution levels of the 3D DWT, using zerotrees. Our scheme requires an efficient bit allocation to assign a bit rate to each subband of the wavelet decomposition, while minimizing the overall distortion. Zerotrees based methods are simpler but sensitive to the propagation of errors through the resolution levels.
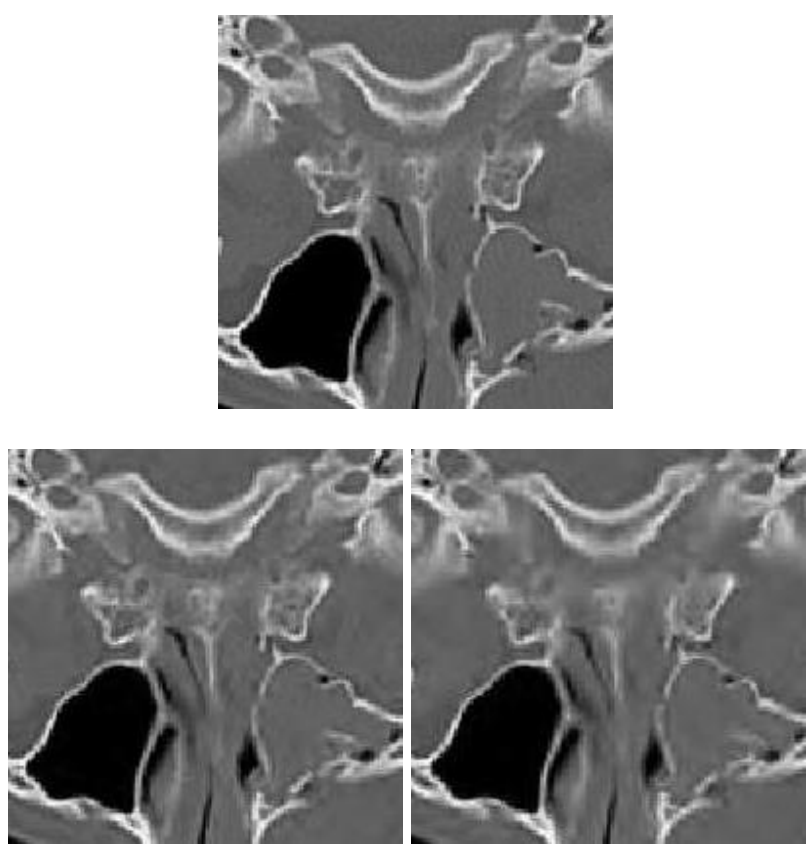

Figure 6: Zoom of decoded slice 60 (Skull) when the target decoding bit rate is $0.1 \mathrm{bpv}$ for the whole 128-slice unit. Clock-wise from the top to the bottom left: Original slice, 3D SPIHT, 3D DZLVQ.

The estimation of the computational complexity of the algorithms used in this work is not a trivial task. Indeed, 3D SPIHT accounts the dependencies between the wavelet subbands which are exploited through zerotrees. The computational complexity (in operations/voxel) of such a structure cannot be expressed. On the other hand, contrary to 3D SPIHT, our proposed 3D DZLVQ algorithm is based on an intraband approach and requires consequently a bit allocation procedure which increases the whole complexity, making 3D DZLVQ a slower algorithm than 3D SPIHT. However, thanks to the proposed analytical solution to the bit allocation problem, the reduction of the complexity is significant. For the medical image data set and the range of bit rates used in the paper, we can show experimentally that the whole computational complexity of 3D DZLVQ falls down to about 66 operations/voxel. The use of our method is thus realistic in a medical data compression application. Furthermore, it is highly parallelizable due to the intraband characteristic.

Our method achieves lossy compression competitive with the best results published in the literature in terms of PSNR. Moreover, it produces reconstructed volumetric medical images visually better than 3D SPIHT, which is known as one of the most efficient methods for this kind of images. This seems to be due to the combination of several points. First, the vector orientation along the details within each subband allows to better capture clusters carrying significant information. In addition, the $L^{1}$ norm of vectors seems to be an appropriate criterion to discriminate significant information from non significant one. Finally, the proposed pyramidal deadzone allows to threshold non significant vectors increasing thus the 
amount of vectors quantized by the null vector, which is advantageously exploited by our entropy coded 3D DZLVQ algorithm. By this way, it is possible to decrease the overall distortion at a constant rate or equivalently to decrease the bit rate at a constant distortion. The improvement of the rate-distortion trade-off can be reached in most of the wavelet subbands, especially in the high and average resolutions of the wavelet decomposition, which yields a better overall visual quality and the preservation of small details.

In order to be fair in the performance evaluation, it is necessary to take into account an important issue. It is well-known that the PSNR is a global criterion which is not sufficient to measure the quality of the reconstructed images, especially in medical applications where the evaluation should be done by experienced radiologists over a large data set. Unfortunately, such studies are very expensive in terms of time and human means. This is the reason why, at our knowledge, none recent works on the actual 3D lossy coders of the literature uses a medical opinion.

Our goal in this paper is mainly to present a new lossy compression algorithm dedicated to the specificities of volumetric medical images. First results reported here are promising as they show gains in terms of PSNR with respect to the best coders published in the literature. Furthermore, the proposed algorithm seems to improve the overall visual quality by producing less blurred reconstructed images at low rates. As mentioned previously, these results should be evaluated by medical experts in terms of visual quality. However, note that first results of another study on compression of 12 bit ENT CTs we drive with two medical experts of the Centre Alexis Vautrin of Nancy - France (centre specialized in cancerology) confirm the superiority of the proposed method over 3D SPIHT for this kind of images. Thus, the next step of our work will include an evaluation of observer performance with medical experts at a larger scale.

\section{CONCLUSION}

We have presented a new 3D medical image compression method based on a multidimensional dead zone which achieves lossy compression competitive with the best results published in the literature in terms of PSNR. By assigning more bits on significant vectors according to a norm-based criterion, our method allows to better preserve fine structures and produces a better overall quality of the reconstructed images which is of importance in medical applications.

\section{REFERENCES}

[1] Raffy P , Gaudeau Y, Miller D, Moureaux J-M, Castellino R.A, "Effect of 3D Wavelet Image Compression on Computer Aided Detection (CAD) Lung Nodule Volumetry" Academic Radiology, 13, issue 10, pp. 1194-1203, 2006.
[2] M Penedo et al, "Free-response receiver operating characteristic evaluation of lossy JPEG2000 and object-based set partitioning in hierarchical trees compression of digitized mammograms “, Radiology, vol. 237 , pp. 450-457, 2005.

[3] Bilgin A., Zweig G, and Marcellin M.W, "ThreeDimensional Image Compression with Integer Wavelet Transforms," Applied Optics 39, pp. 1799-1814, 2000.

[4] Benoit-Cattin H, Baskurt A, Prost R, "3D medical image coding using separable 3D wavelet decomposition and lattice vector quantization,” Signal Processing 59, pp. 139-153, 1997

[5] Gray R. M., Neuhoff D., “Quantization,” IEEE Trans. on Information Theory 44, pp. 1-63, 1998.

[6] Barlaud M., Solé P., Gaidon T., Antonini M., Mathieu P., "Pyramidal lattice vector quantization for multiscale image coding,” IEEE Trans. Image Processing 3, pp. 367-381, 1994.

[7] Voinson, T, Guillemot, L, Moureaux J-M, Image "compression using lattice vector quantization with code book shape adapted thresholding," ICIP, pp. 641-644, Rochester, USA, 2002.

[8] Fischer T, “A pyramid vector quantizer,” IEEE Transactions Information Theory 32, pp. 569-583, 1986.

[9] Guillemot L, Gaudeau Y, Moureaux J-M, “A new fast bit allocation procedure for image coding based on wavelet transform and dead zone lattice vector quantization," ICIP, Genoa, Italy, 2005.

[10] Kim YS, Pearlman WA, "Lossless Volumetric Image," Compression. Proceedings of SPIE, Applications of Digital Image Processing XXII, pp. 305-312, 1999.

[11] Cho S.,Kim.D, Pearlman W.A, "Lossless Compression of Volumetric Medical Images with Improved Three-Dimensional SPIHT Algorithm,” Journal of Digital Imaging 17, pp. 57-63, 2004.

[12] Xiong Z, Wu X., Cheng S, Hau J, "Lossy-to-Lossless Compression of Medical Volumetric Data Using ThreeDimensional Integer Wavelet Transform," IEEE Trans. on Medical Imaging 22, pp. 459-470, 2003.

\section{ACKNOWLEDGMENTS}

The authors would like to thank Drs Philippe Henrot and Pierre Graff of the Centre Alexis Vautrin (Nancy-France) for helpful discussions related to the evaluation of lossy compressed medical images. This work has been supported by AGIR project (http://www.aci-agir.org) funded by the ACI Masses de Données program of the French Ministry of Research. 